\title{
Circadian rhythm of interictal epileptiform discharges and changes of spindles in patients with temporal lobe epilepsy.
}

\author{
Yun-Li Yu*, Yong-Li Yan, Su-Fen Tian, Zhan-Hui Feng, Meng-Ting Shi \\ Department of Neurology, Affiliated Hospital of Guizhou Medical University, Guizhou Guiyang 550004, PR China
}

\begin{abstract}
Background: This study aims to explore the circadian rhythm of interictal epileptiform discharges (IEDs) and changes of spindles in patients with temporal lobe epilepsy (TLE).

Methods: Subjects were divided into two groups: research group (patients diagnosed with TLE, $n=50$ ) and control group $(n=30)$. The whole-night natural sleep of all subjects was monitored, analysis and statistics were performed on IED, and the number, amplitude and the average duration of spindles were compared between these two groups.

Results: (1) In TLE patients, the number of IED was larger in non-rapid eye movement (NREM) stages $1+2$ and NREM stages $3+4$ than in the waking stage and rapid eye movement (REM) stage, and the differences were statistically significant $(P<0.05)$. (2) TLE patients had bilateral asymmetric sleep spindles, and their amplitude was lower in the affected side than in the unaffected side. Furthermore, the number, amplitude and mean duration of sleep spindles in the affected side in NREM stage 2 was significantly reduced compared with that in the control group $(\mathbf{P}<0.05)$.

Conclusions: (1) TLE patients have the highest frequency of IED in slow wave sleep, followed by NREM stages 1+2, waking and REM stages. Slow wave sleep easily induces IED. (2) The number, amplitude and mean duration of sleep spindles in the affected side in NREM stage 2 were significantly reduced, compared with that in the control group. This suggests that changes in spindles have a lateralization value.
\end{abstract}

Keywords: Temporal lobe epilepsy, Sleep, Polysomnography, Sleep spindles, Attack period between epileptic discharge.

Abbreviations:

IEDs: Interictal Epileptiform Discharges; TLE: Temporal Lobe Epilepsy; REM: Rapid Eye Movement; NREM: Non-rapid Eye
Movement; VEGEG: Video Electroencephalogram; PSG: Polysomnography; GABA: Gamma Aminobutyric Acid

Accepted on December 30, 2017

\section{Introduction}

Temporal lobe epilepsy (TLE) is the most common intractable epilepsy. TLE patients are often accompanied by sleep disorders. It has been reported that the incidence of insomnia in patients with TLE is $52 \%$, while the incidence of daytime somnolence is as high as $70 \%[1,2]$. More and more attention has been given to the relationship between sleep and epilepsy, which are mutually influenced and closely related with each other. In recent years, long-range video electroencephalogram (VEEG) and polysomnography (PSG) concurrently form the "VEEG-PSG" technology, which is more accurate in judging the relationship between sleep and epilepsy. Therefore, this method was used in this study to investigate the relationship between the waking-sleep rhythm in TLE and IED, and explore the characteristics of sleep spindles in TLE. This would provide evidence for further studies on the structural features of sleep disorder, and add to the indicators for localization of epileptic foci in TLE.

\section{Materials and Methods}

\section{Subjects}

From September 2014 to February 2016, 50 TLE patients diagnosed in the Outpatient Department and Neurological Inpatient Department of the Affiliated Hospital of Guizhou Medical University were enrolled into this study. All patients had more than three follow-ups. These patients were divided into two groups: intractable group, which comprised of 16 patients with an average age of 32 years old; tractable group, which comprised of 34 patients with an average age of 29 years old. Among these 50 patients, 24 patients were male and 26 patients were female; and the age of these patients ranged between 17-50 years old. Furthermore, the course of the disease ranged between 5-30 years. The age of initial onset ranged between 0.5-45 years old. All of the above patients were received by the Epilepsy Clinic, Department of Neurology, Affiliated Hospital of Guizhou Medical University, 
by deputy chief physicians or senior experts, in accordance with the commonly used TLE diagnostic criteria [3]. Before whole-night sleep monitoring, all patients underwent head computed tomography (CT) or magnetic resonance imaging (MRI); and some patients additionally underwent magnetic resonance blood-flow scanning (MRS) and positron emission tomography-computed tomography (PET-CT). No clinical seizure occurred during the whole-night monitoring. Imaging findings: unilateral temporal lesions in 21 cases (manifestations: hippocampal sclerosis in 12 cases, temporal horn enhancement combined with hippocampal atrophy in five cases, vascular malformation in right temporal lobe combined with hemorrhage in one case, cavernous hemangioma in two cases, and temporal lobe dysplasia in one case), bilateral hippocampal sclerosis in seven cases, post-temporal-lobectomy manifestations in two cases, white matter ischemic lesions in three cases, and bilateral enlarged Virchow-Robin spaces in one case. The remaining 16 cases did not reveal any abnormality.

Past history: Two cases had a history of meningitis, and two cases had a history of febrile convulsions. Through head MRI, patients who had other causes of epilepsy such as tumors and cortical dysplasia were excluded from this study. In addition, 30 healthy persons with normal physical examination results were enrolled as controls. Among these subjects, 16 subjects were male and 14 subjects were female; and their age ranged between 17-50 years old (average age: 33 years old). All subjects had no neurological or psychiatric history, no intracranial organic, traumatic diseases, or other diseases that may affect the structure or function of the brain. Neurological examination revealed normal results.

\section{Methods}

Electrode placement: Video electroencephalogram (VEEG) and polysomnography (PSG) monitoring module: All TLE patients and controls were monitored by VEEG-PSG modules (Nicolet, USA): electrodes were placed according to the international 10-20 system, and 21-lead EEG monitoring was performed (including sphenoidal electrode, for recording the two states of waking and sleeping), in order to monitor the whole-night natural sleep. EEG examination results were drawn by two physicians who had worked in the EEG room for more than two years, and had an EEG intermediate professional title. Sleep stage was judged by the addition of eye movement, mandibular electromyography (EMG) and ECG.

Proportion and number of TLE patients with IED in each period: The proportion of TLE patients with IED in each stage refers to the proportion of TLE patients that presented with IED in the waking and sleep stages. IED counting method: The EEGs of the waking stage and the following four stages: REM stage, NREM stages $1+2$, and NREM stages $3+4$ in the first two sleep cycles were selected for the manual counting of IED. The counting method was as follows: the EEG-PSG graphics of a total of 40 periods $(30 \mathrm{~s}$ per period, the total time was 20 $\min )$ in the above stages were selected for analysis. If the periods in the cycles were not enough, the next cycle was included for analysis. Evaluation criteria for interictal epileptiform discharges [4]: (1) Epileptiform discharge waves should be clearly identified from the background. (2) The waveform is sharp, and the ascending and descending branches are not symmetrical. The sharp wave duration was $\leq 200 \mathrm{~ms}$, a discharge time of $<70 \mathrm{~ms}$ was defined as a spike wave, and 70-200 ms was defined as a sharp wave, spreading to adjacent leads. The occurrence of spike clusters was recorded as an event. (3) In this study, sharp waves, spikes, spike slow waves, sharp slow waves and multiple spikes appeared. The distribution of epileptiform discharges, such as sharp wave, sharp slow wave and spike wave in the waking stage, NREM stages $1+2$, NREM stages $3+4$ and REM stage were manually calculated; and the number of epileptiform discharges in each stage was calculated stage by stage.

\section{Selection of sleep spindles:}

(1) Sleep spindle number: The 20 min with the most frequent sleep spindles in NREM stage 2 in the first complete sleep cycle of the whole-night sleep was selected, and the number of spindles was counted screen by screen. Sleep spindle criteria were as follows: frequency range was between $11-15 \mathrm{~Hz}$, and the waves are the characteristic brain waves in N2 sleep, which mainly appears in the central area of the skull; the amplitude increases gradually, and gradually reduces thereafter; most of the waves have a frequency of $12-14 \mathrm{~Hz}$, and the duration of each wave is $>0.5 \mathrm{~s}$.

(2) Sleep spindle amplitude: The vertical height of spindles in leads $\mathrm{C} 3$ and $\mathrm{C} 4$ were measured using the EEG measurement gauge.

(3) The average duration of sleep spindles: the ratio of the total duration of sleep spindles to the spindle number within the 20 min selected in this study.

\section{Data statistics}

Data were managed using Excel software. SPSS 19.0 statistical software was used for the statistical analysis of data. The measurement data in the above examination is presented as mean \pm standard deviation when normally distributed. The means of these multiple samples were compared using univariate analysis of variance. Since the numbers of interictal epileptiform discharges were not normally distributed, these data were converted into normally distributed data using the following method: the original value +1 . Then, the logarithm of the sum was calculated. Next, these calculated results were statistically processed. $\mathrm{P}<0.05$ was considered statistically significant.

\section{Results}

\section{Comparison of discharge times of during each stage of waking-sleep cycle in TLE patients}

The proportion of patients with IED varied in different stages, the three patients had IEDs that only appeared during the 
waking period, and the proportion of IEDs during the waking stage was 6\% (3/50). Furthermore, 20 patients had IEDs that only appeared in the sleep stage, and the proportion of IED was $40 \%(20 / 50)$ in the sleep stage. In addition, 27 patients had IEDs that appeared during the waking and sleeping stages; and the proportion of IEDs during the waking and sleep stages was $54 \%(27 / 50)$. The number of IEDs in TLE patients in NREM stages 1+2 (median: 36) and NREM stages 3+4 (median: 46) was larger than that in the waking stage (median: 14) and REM stage (median: 2.5), and the differences were statistically significant $(\mathrm{P}<0.05)$. The difference in the number of IEDs between NREM stages 1-2 and NREM stages 3-4 was not statistically significant $(\mathrm{P}>0.05)$. The discharge times in the waking stage were more than the discharge times in the REM stage, and the difference was statistically significant $(\mathrm{P}<0.05)$.

\section{Comparison of sleep spindles}

Adacchi et al. defined the lateralization of IEDs as the side that had unilateral IEDs exceeding 75\% was the dominant side [5]. In this study, according to the side of the IED, the TLE group was divided into two groups: unilateral discharge group and bilateral discharge group. TLE patients with unilateral discharge were subjected to the further study of spindles, and the discharge side was defined as the affected side. Among these patients, abnormal discharges in the five TLE patients had bilateral origin, and the amplitudes of spindles were bilaterally symmetrical in 10 TLE patients. Hence, these 15 patients were excluded. Therefore, the number of subjects was 35 in TLE group and 30 in the control group.

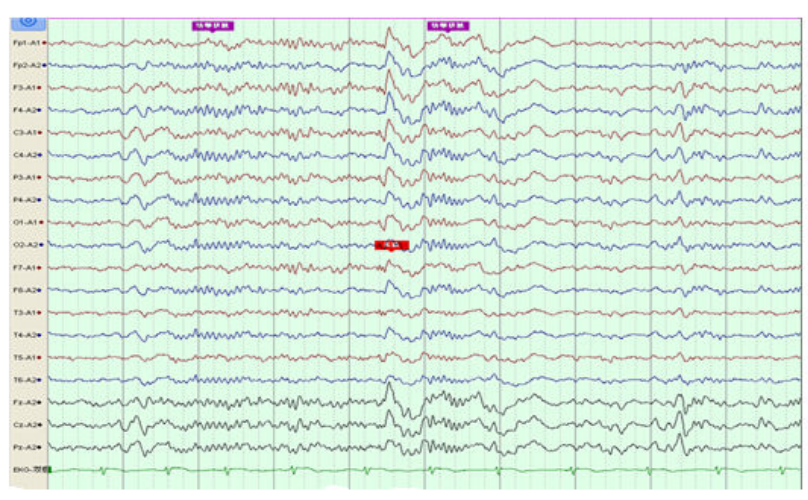

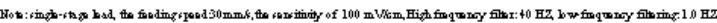

Figure 1. Male, 26 years old, TLE patients with discharge in the anterior and middle of the left temporal, the amplitude of spindles in the left was lower than that in the right side and the duration of it was shorter.

Compared with the control group, sleep spindles in TLE patients were poorly differentiated in morphology, relatively sparse and had shorter durations, and presented with bilateral asymmetry in the EEG of 35 patients (Figure 1). Furthermore, the amplitude was significantly lower in the affected side than in the healthy side and both sides of patients in the control group $(52.26 \pm 6.33$ vs. $57.03 \pm 6.24 ; 52.26 \pm 6.33$ vs. $59.97 \pm$ 5.92), and the differences between these two groups was statistically significant $(\mathrm{P}<0.05)$. Compared with the control group, the number of sleep spindles in patients with TLE was significantly reduced $(75.30 \pm 37.23$ vs. $89.60 \pm 24.71)$; and the differences between these two groups were statistically significant $(\mathrm{P}<0.05)$. The mean duration of spindles was shortened in TLE patients compared with controls (Figure 2). This reduced from the normal level of 0.81 second to 0.74 second, and the difference between these two groups were statistically significant $(\mathrm{P}<\mathrm{O} .05$, Table 1$)$.

Table 1. Comparison of sleep spindles between TLE group and control group.

\begin{tabular}{|c|c|c|c|c|c|}
\hline \multirow{2}{*}{$\begin{array}{l}\text { Spindles } \\
\text { Wave }\end{array}$} & \multirow{2}{*}{$\begin{array}{l}\text { The control } \\
\text { group }\end{array}$} & \multicolumn{2}{|c|}{ TLE group } & \multirow[t]{2}{*}{$\mathbf{F}$} & \multirow[t]{2}{*}{ p } \\
\hline & & Health side & III side & & \\
\hline The number & $89.60 \pm 24.71$ & $75.30 \pm 37.23^{\mathbf{\Lambda}}$ & $\begin{array}{l}75.30 \\
37.23^{\Delta}\end{array}$ & \pm 2.063 & $<0.05$ \\
\hline $\begin{array}{l}\text { Amplitude } \\
(\mu \mathrm{V})\end{array}$ & $59.97 \pm 5.92$ & $57.03 \pm 6.24$ & $\begin{array}{l}52.26 \\
6.33^{\Delta} \Delta\end{array}$ & \pm 13.02 & $<0.05$ \\
\hline $\begin{array}{l}\text { The } \\
\text { average } \\
\text { duration (s) }\end{array}$ & $0.81 \pm 0.08$ & $0.78 \pm 0.09$ & $0.74 \pm 0.08^{\Delta}$ & 4.52 & $<0.05$ \\
\hline
\end{tabular}

Note: $\triangle$ Show with the control group comparison, $p<0.05 ;{ }^{\Delta}$ Representation with the contralateral contrast comparison, $\mathrm{p}<0.05$.

\section{Discussion}

The relationship between epilepsy and sleep has been found for a long time. These two are closely related to each other. The whole-night video EEG monitoring includes a normal sleep cycle. Therefore, it does not need to use inducing tests such as sleep deprivation. This new technology can better find the distribution of IEDs in each stage of sleep. Sleep structure can be analyzed in detail by PSG. The combined application of these two electrophysiological examination techniques can better determine the relationship between IED and sleep structure and spindles.

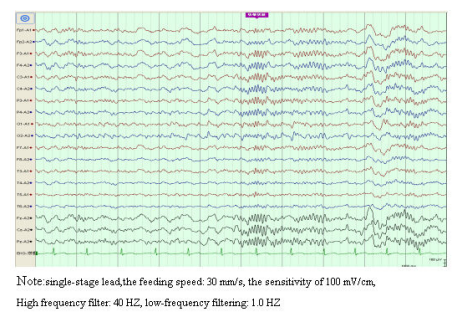

Figure 2. Male, 23 years old, sleep spindles in bilateral were symmetrical in normal control group.

\section{The distribution and significance of IED in the waking stage and different stages of sleep in TLE patients and its significance}

The biological rhythm of the human body has the periodicity of awakening and sleeping. Brainstem reticular formation plays an important role in the mechanism that cause awakening and sleep. The activation effects of the ascending brainstem 
reticular activating system on the brain cortex and limbic system decrease during sleep, making the abnormal electrical activity of the brain easy to release and activate. Studies [5-7] have revealed that the positive rate of IEDs was $20.6 \%$ in the waking stage, $40.4 \%$ in the sleep stage, and $38.9 \%$ in the waking and sleeping stages. The positive rate of IED was higher in the sleep stage than that in the waking stage. In this study, the proportion of IEDs was $6 \%(3 / 50)$ in the waking stage and $40 \%(20 / 50)$ in the sleep stage. Furthermore, the proportion of IEDs was 54\% (27/50) in the waking and sleep stages. This was consistent with the results of previous studies. In general, IEDs in patients with epilepsy was higher in the sleep stage compared with the awakening stage. Malow et al. reported that [8] NREM sleep depth in TLE patients is very important for the activation of IED. IED in TLE patients was mainly found in the NREM stages, and was more commonly found in the SWS sleep of NREM sleep; which was consistent with the results of this study. This may be related to the reduction of brainstem reticular formation activity, decreased inhibition of cortical neurons, and the easy activation of IEDs in epilepsy patients in SWS sleep. Sammaritano [9] revealed that in a study comprised of 31 subjects with TLE, IEDs significantly increased in SWS sleep, compared with the shallow sleep stage of NREM, REM sleep stage and waking stage. Frequent IEDs may be an important factor for the disorder of sleep structure in TLE patients. Among these, part of the TLE patients underwent intracranial electrodes. These results also revealed that SWS preferentially activate IED. IED activated by sleep can provide useful clinical information for preoperative localization in patients with intractable epilepsy. In this study, natural sleep was divided into four stages: waking stage, NREM stage $1+2$, NREM stage $3+4$, and REM stage. The number of IEDs was largest in NREM stage $1+2$ and NREM stage $3+4$, and this number was lowest in the REM stage and waking stage. This result is similar to that of the study of Sammaritano. Due to the increase in reticular formation activity and the desynchronization of electrical activity of the brain in REM sleep, the release and dissemination of the abnormal electrical activity of the brain may be suppressed in this stage; causing the lower probability of IEDs in REM sleep. This was consistent with the results of the present study. However, this does not suggested that there was no abnormal release brain electrical activity in REM sleep. Studies have revealed that for patients with bilateral spikes and slow waves in the EEG during the waking stage, the spike waves were released from only one side in the REM stage. IEDs in the REM stage would be relatively limited. The value of IEDs for the localization of the seizure foci was more significant in the REM stage than that in the NREM stage $[10,11]$.

\section{Change of sleep spindles and its significance}

Sleep spindles derive from the rhythmic depolarization of thalamic reticular nucleus neurons. The rhythmic activity of thalamic reticular neurons can induce the rhythmic discharge of thalamic cortical neurons. The latter would cause cortical neurons to produce a synchronized post-synaptic potential.
This potential is the sleep spindles revealed by sleep EEG. Research on the neurotransmitter in TLE patients revealed that disorders in neurotransmitter levels were found in most of patients with epilepsy. The manifestations are mainly low concentrations of gamma aminobutyric acid (GABA). GABA is an important mediator produced in sleep spindles. A decrease in the amount of GABA can affect the formation of sleep spindles [12], while the increase of Ach and NE would inhibit sleep spindles. A study revealed that for patients with partial seizures, the number and power spectrum of sleep spindles were significantly lower before the seizures, compared with baseline levels in the interval [13]. Some studies concluded that the decrease in sleep spindles was a herald to seizures. During video EEG monitoring, the significant decrease in sleep spindles may be used as a warning signal of the secondary generalization to systemic seizures. The number, amplitude and mean duration of sleep spindles in patients in the TLE group were significantly lower compared with that in the control group. This is probably an important reason that TLE patients would wake up from sleep as long as any slight noise appears. Compared with the control group, in the 50 epilepsy patients with IED, the occurrence rate of spindle wave asymmetry in sleep was $70 \%(35 / 50)$; and sleep spindle wave symmetry was considered an important factor for maintaining the stability of sleep and in inhibiting IED [14]. A study revealed that $\mathrm{VEEG}+$ sphenoidal electrodes had high lateralization accuracy. MRI and VEEG+sphenoidal electrodes was the most commonly used method for the localization of epileptogenic focus, and the consistent results of these two enabled the localization of epileptic foci [15]. The affected side sleep spindles with unilateral interictal discharge have decreased amplitudes and wave number, as well as a shortened duration; suggesting that changes in spindles have a lateralization value [16].

\section{Conclusion}

In this study, it was found that TLE patients often presented with bilateral spindle wave asymmetry. This suggests that attention should be given to changes in sleep spindles in future clinical work, since it may have a certain value for the lateralization of epileptic foci. However, the application value of these spindles for the lateralization of the epileptic focus has rarely been reported. As it is known, sleep easily induces IED, and sleep spindle wave symmetry is considered an important factor in maintaining the stability of sleep and inhibiting IED. In other words, sleep spindle asymmetry may be one of the causes of IED. The size of samples in this study is relatively small. Hence, this finding needs to be confirmed through largesample studies in the future.

\section{References}

1. Khatami R, Zutter D, Siegel A, Mathis J, Donati F, Bassetti CL. Sleep-wake habits and disorders in a series of 100 adult epilepsy patients-a prospective study. Seizure 2006; 15: 299-306. 
2. Maganti R, Hausman N, Koehn M, Sandok E, Glurich I, Mukesh BN. Excessive daytime sleepiness and sleep complaints among children with epilepsy. Epilepsy Behav 2006; 8: 272-277.

3. Panayiotopoulos CP. The epilepsies seizures, syndromes and management. Bladon Medical Publishing 2006; Oxfordshire, UK.

4. Ferrillo F, Beelke M, De Carli F, Cossu M, Munari C. Sleep-EEG modulation of interietalepil eptiform discharges in adult partial epilepsy: a spectral analysis study. Clin Neurophysiol 2000; 111: 916-923.

5. Adachi N, Alarcon G, Binnie CD, Elwes RD, Polkey CE, Reynolds EH. Predietive value of interictal epileptiform discharges during non-REM sleep on scale EEG recordings for the lateralization of epileptogenesis. Epilepsia 1998; 39: 628-632.

6. Lu YD, Chu FN, Meng HM, Cui L, Wang Z. The relationship between interictal epileptic discharges and sleep cycle of 240 epilepsy patients. Chin J Neurol 2014; 47: 35-38.

7. Wang F, Pan SP, Liu XZ, Wang MY, He J, Hu JY. A study on circadian rhythm of interictal epileptiform discharges in patients with localization related epilepsy. Chin J Behav Med Brain Sci 2013; 22: 406-408.

8. Malow BA, Lin X, Kushwaha R, Aldrich MS. Interictal spiking increases with sleep depth in temporal lobe epilepsy. Epilepsia 1998; 39: 1309-1316.

9. Sammaritano M, Gigli GL, Gotman J. Interictal spiking during wakefulness and sleep and the localization of foci in temporal lobe epilepsy. Neurology 1991; 41: 29-37.
10. Lucey BP, Noetzel MJ, Duntley SP. Arousals and myoelonic movements associated with interictal epileptiform discharges in NREM and REM sleep. Clin Neurol Neurosurg 2011; 113: 419-422.

11. Busek P, Buskova J, Nevsimalova S. Interictal epileptiform discharges and phasic phenomena of REM sleep. Epileptic Disorder 2010; 12: 217-221.

12. Wu X. Epilepsy and seizure disorders. People's Military Medical Publisher 2001; BeiJing, China.

13. Tezer FI, Remi J, Erbil N, Noachtar S, Saygi S. A reduction of sleep spindles heralds seizures in focal epilepsy. Clin Neurophysiol 2014; 125: 2207-2211.

14. Dang Vu TT, McKinney SM, Buxton OM, Solet JM, Ellenbogen JM. Spontaneous brain rhythms predict sleep stability in the face of noise. Curr Biol 2010; 20: 626-627.

15. Cheng Q, Zhai GD, Pang Q. A clinical study of the accuracy of location methods for temporal lobe seizure forci. Chin J Neurosurg Dis Res 2010; 9: 393-396.

16. Nobili L, Baglietto GM, Beelke EM, Ferrillo F. Spindlesinducing mechanism modulates sleep activation of interictal epileptiform discharges in the Landau-Kleffner syndrome. Epilepsia 2000; 41: 201-206.

\section{*Correspondence to}

Yun-Li Yu

Department of Neurology

Affiliated Hospital of Guizhou Medical University

PR China 